\title{
Poincaré-Cartan integral invariant and canonical transformations for singular lagrangians
}

\author{
D. Dominici \\ Istituto Nazionale di Fisica Nucleare, Sezione di Firenze Istituto di Fisica Teorica dell'Università di Firenze, \\ Firenze, Italia \\ J. Gomis \\ Department de Física Teòrica, Universitat de Barcelona, Barcelona, Spain
}

(Received 7 May 1979; accepted for publication 30 January 1980)

\begin{abstract}
In this work we develop the canonical formalism for constrained systems with a finite number of degrees of freedom by making use of the Poincaré-Cartan integral invariant method. A set of variables suitable for the reduction to the physical ones can be obtained by means of a canonical transformation. From the invariance of the Poincaré-Cartan integral under canonical transformations we get the form of the equations of motion for the physical variables of the system.
\end{abstract}

\section{INTRODUCTION}

It is known that many interesting physical systems are described by singular Lagrangian. Some examples are provided by the electromagnetic, the gravitational, the YangMills fields, and some relativistic models. ${ }^{1}$ Features of all these theories are the invariance under certain transformations and the presence of relations (constraints) among the canonical variables, which restrict the motion to a hypersurface of the phase space.

A method for developing the canonical formalism and the quantization of constrained systems was proposed by Dirac. ${ }^{2}$ The constraints are classified into two groups (firstclass and second-class), depending on their algebraic properties with respect to Poisson brackets. The dynamics of the system is generated by an extended Hamiltonian, obtained by adding a linear combination of first-class constraints to the canonical one. One must take into account the presence of second-class constraints by working with generalized Poisson brackets (Dirac brackets). The problem of the quantization is complicated by the search for a set of variables independent and canonical with respect to Dirac brackets. Instead of following the Dirac technique, these variables can be directly obtained, as suggested by Shanmugadhasan, ${ }^{3}$ as a subset of the variables of a canonical transformation, whose existence is based on some theorems on involutory systems..$^{45}$ and function groups. ${ }^{6}$ We want to stress that this method, as well as the Dirac brackets technique, is a local one; in fact the existence of the canonical transformation is only locally guaranteed. ${ }^{4}$

In this work we pursue the study of the extension of the formalism of the Poincaré-Cartan integral invariant to constrained systems with a finite number of degrees of freedom, which one of us began in Ref. 7, and making use of the invariance of the Poincaré-Cartan integral under canonical transformations, the equations of motion for a set of variables free with respect to second-class constraints are easily obtained. Furthermore, working in this reduced space of the variables independent with respect to second-class constraints, a canonical transformation which isolates the gauge-indepen- dent variables from the gauge-dependent ones is performed. This is the great advantage of this technique with respect to the Dirac one. An interesting result is that, for Lagrangians homogeneous of first-degree in the velocities, this procedure corresponds to the Hamilton-Jacobi method.

In Sec. 2 we review and extend the Poincaré-Cartan integral formalism for constrained systems. Section 3 is devoted to the introduction of the concept of canonical transformation and to the proof of the invariance of the PoincaréCartan integral under canonical transformations. In Sec. 4 we perform the canonical transformation extended to the second-class constraints and the Hamilton equations for the new variables are obtained. In Sec. 5 the Hamilton equations for the set of variables free with respect to first- and secondclass constraints are obtained.

\section{POINCARÉ-CARTAN INTEGRAL INVARIANT FOR CONSTRAINED SYSTEMS}

The Poincaré-Cartan integral invariant plays a fundamental role in standard classical mechanics since, from its invariance, it follows that the equations of motion of the dynamical system are Hamilton canonical equations. ${ }^{8}$

In Ref. 7 this result was generalized to systems described by singular Lagrangians.

Let us now review the essential points of this generalization. Let us consider a dynamical system described by a singular Lagrangian

$$
L=L\left(q_{s}, \dot{q}_{s}, t\right), \quad(s=1, \ldots, n) .
$$

Due to the singularity of the Lagrangian, the motion of the system is restricted to a hypersurface of the phase space, determined by a set of constraints. Let

$$
\Omega_{\alpha}\left(q_{s}, p_{s}\right)=0, \quad(\alpha=1, \ldots, T-W),
$$

be first-class constraints and

$$
\Omega_{\beta}\left(q_{s}, p_{s}\right)=0, \quad(\beta=T-W+1, \ldots, T)
$$

be second-class.

Making a general variation of the action 


$$
W=\int_{t_{0}}^{t_{1}} d t L,
$$

it is possible to show that the integral

$$
I=\oint_{C}\left(p_{s} \delta q_{s}-H_{c} \delta t\right)
$$

calculated along an arbitrarty closed contour lying on the hypersurface $S$ of the extended phase space $\left(q_{s}, p_{s}, t\right)$, defined by Eqs. (2.2) and (2.3), is invariant under an arbitrary displacement (with deformation) of the contour along any tube of dynamical trajectories. $H_{c}$ is the canonical Hamiltonian of the system. $I$ is called the Poincaré-Cartan integral invariant.

Let us now review the proof of the following theorem with some details.

Theorem 1: Let us suppose to have a dynamical system, constrained by Eqs. (2.2) and (2.3), whose trajectories satisfy a system of first order differential equations involving arbitrary functions $l_{\alpha}(\alpha=1, \ldots, T-W)$

$\frac{d}{d t} q_{s} \approx f_{s}\left(q_{s}, p_{s}, t ., l_{\alpha}\right) \quad \frac{d}{d t} p_{s} \approx g_{s}\left(q_{s}, p_{s}, t, l_{s}\right)$,

where the sign $\approx$ (weak equality) means equality on the hypersurface $S$ [defined by Eqs. (2.2) and (2.3)]. Let $H_{c}$ be a function with the property

$$
\left\{\Omega_{\alpha}, H_{c}\right\} \approx 0 \text {. }
$$

Then, the necessary and sufficient for Eqs. (2.6) be Hamilton equations is that the Poincaré-Cartan integral (2.5) be invariant.

Proof: Firstly, see that the invariance of the PoincaréCartan integral is a sufficient condition.

Following the book of Gantmacher, we introduce an auxiliary variable $\mu$, supplementing Eq. (2.6) with one more equation

$$
\frac{d q_{1}}{f_{1}}=\cdots=\frac{d q_{n}}{f_{n}}=\frac{d p_{1}}{g_{1}}=\cdots=\frac{d p_{n}}{g_{n}}=d t=\pi d \mu,
$$

$\pi$ being an arbitrary function in the extended phase space. For each determination of the $l_{\alpha}$ 's we find, integrating Eqs. (2.8),

$$
\left\{\begin{array}{l}
q_{s}=q_{s}\left(\mu ; q_{s}^{0}, p_{s}^{0}, t_{0}\right) \\
p_{s}=p_{s}\left(\mu ; q_{s}^{0}, p_{s}^{0}, t_{0}\right), \\
t=t\left(\mu ; q_{s}^{0}, p_{s}^{0}, t_{0}\right)
\end{array}\right.
$$

where $q_{s}^{0}, p_{s}^{0}, t_{0}$ are the initial values, corresponding to $\mu=0$, which lie on the hypersurface $S$. In order to obtain a tube of dynamical trajectories (2.9), we choose the initial points on a closed curve, parametrized by means of $\alpha$, contained in $S$.

The parametric equations for the dynamical paths that form the tube are

$$
q_{s}=q_{s}(\mu, \alpha), \quad p_{s}=p_{s}(\mu, \alpha), \quad t=t(\mu, \alpha) \quad(0 \leqslant \alpha \leqslant l) .
$$

The value of $\alpha$ isolates a generatrix of the tube while $\mu$ fixes a definite point on this generatrix. Assuming $\mu=$ const, Eqs. (2.10) define a closed curve embracing the tube; by calculating the integral along it, we get $I=I(\mu)$.
If we agree that $d$ means differentiation with respect to $\mu$ and $\delta$ with respect to $\alpha$, by invariance we have

$d I=\oint\left[d p_{s} \delta q_{s}+p_{s} d \delta q_{s}-d H_{c} \delta t-H_{c} d \delta t\right]=0$.

Integrating by parts, dividing by $d \mu=d t / \pi$ and using Eqs. (2.6) we get

$$
\begin{gathered}
\oint\left\{\left(g_{s}+\frac{\partial H_{c}}{\partial q_{s}}\right) \delta q_{s}+\left(-f_{s}+\frac{\partial H_{c}}{\partial p_{s}}\right) \delta p_{s}\right. \\
\left.+\left(-\frac{d H_{c}}{d t}+\frac{\partial H_{c}}{\partial t}\right) \delta t\right\} \pi=0 .
\end{gathered}
$$

Since $\pi$ is an arbitrary factor we obtain

$$
\begin{aligned}
& \left(g_{s}+\frac{\partial H_{c}}{\partial q_{s}}\right) \delta q_{s}+\left(-f_{s}+\frac{\partial H_{c}}{\partial p_{s}} \delta p_{s}\right) \\
& +\left(-\frac{d H_{c}}{d t}+\frac{\partial H_{c}}{\partial t}\right) \delta t \approx 0 .
\end{aligned}
$$

The $\delta q_{s}$ and the $\delta p_{s}$ are not independent, since $C$ must belong to $S$. So they must satisfy

$$
\begin{aligned}
& \frac{\partial \Omega_{\alpha}}{\partial q_{s}} \delta q_{s}+\frac{\partial \Omega_{\alpha}}{\partial p_{s}} \delta p_{s}=0, \\
& \frac{\partial \Omega_{\beta}}{\partial q_{s}} \delta q_{s}+\frac{\partial \Omega_{\beta}}{\partial p_{s}} \delta p_{s}=0 .
\end{aligned}
$$

Introducing a set of Lagrangian multipliers $l_{\alpha}, l_{\beta}$ ( $\alpha=1, \ldots, T-W, \beta=T-W+1, \ldots, T)$, from Eqs. (2.13) and (2.14) we deduce

$$
\begin{gathered}
g_{s} \approx-\frac{\partial H_{c}}{\partial q_{s}}-l_{\alpha} \frac{\partial \Omega_{\alpha}}{\partial q_{s}}-l_{\beta} \frac{\partial \Omega_{\beta}}{\partial q_{s}} \\
f_{s} \approx \frac{\partial H_{c}}{\partial p_{s}}+l_{\alpha} \frac{\partial \Omega_{\alpha}}{\partial p_{s}}+l_{\beta} \frac{\partial \Omega_{\beta}}{\partial p_{s}} .
\end{gathered}
$$

By requiring that the hypersurface be stationary, the $l_{\beta}$ 's can be determined

$$
l_{\beta} \approx c_{\beta \beta^{\prime}}\left\{\Omega_{\beta^{\prime}}, H_{c}\right\},
$$

where

$$
c_{\beta \beta^{\prime}}\left\{\Omega_{\beta^{\prime}}, \Omega_{\beta^{\prime \prime}}\right\} \approx \delta_{B \beta^{\prime \prime}} .
$$

The Eqs. (2.6) can be written in the form

$$
\left\{\begin{array}{l}
\dot{q}_{s} \approx \frac{\partial H}{\partial p_{s}}=\left\{q_{s}, H\right\} \\
\dot{p}_{s} \approx-\frac{\partial H}{\partial q_{s}}=\left\{p_{s}, H\right\},
\end{array}\right.
$$

with

$$
H=H_{c}+l_{\alpha} \Omega_{\alpha}-\Omega_{\beta} c_{\beta \beta^{\prime}}\left\{\Omega_{\beta^{\prime}}, H_{c}\right\} .
$$

By following an analogous reasoning and starting from Eqs. (2.18) and (2.19), it is possible to show the invariance of the Poincaré-Cartan integral (2.5). Thus the proof of the theorem is complete. 


\section{CANONICAL TRNASFORMATIONS AND POINCARÉ- CARTAN INTEGRAL INVARIANT}

Let us now extend the concept of canonical transformation to constrained systems, by introducing, as in standard classical mechanics, the following

Definition: Given a dynamical system, constrained by Eqs. (2.2) and (2.3), whose equations of motion are given by Eq. (2.18), a transformation

$$
Q_{s}=Q_{s}(q, p, t), \quad P_{s}=P_{s}(q, p, t) \quad(s=1, \ldots, n),
$$

is called canonical if a function $K_{c}$ exists so that Eqs. (2.18) become

$$
\dot{Q}_{s} \approx \frac{\partial K}{\partial P_{s}}=\left\{Q_{s}, K\right\}, \quad \dot{P}_{s} \approx-\frac{\partial K}{\partial Q_{s}}=\left\{P_{s}, K\right\},
$$

with

$$
K=K_{c}+l_{\alpha} \tilde{\Omega}_{\alpha}-\tilde{\Omega}_{\beta} \tilde{c}_{\beta \beta^{\prime}}\left[\left\{\tilde{\Omega}_{\beta^{\prime}}, K_{c}\right\}+\frac{\partial \tilde{\Omega}_{\beta^{\prime}}}{\partial t}\right]
$$

and

$$
\tilde{c}_{\beta \beta^{\prime}}\left\{\tilde{\Omega}_{\beta^{\prime}}, \tilde{\Omega}_{\beta^{*}}\right\}=\delta_{\beta \beta^{*}} .
$$

The $\tilde{\Omega}_{\alpha}(Q, P, t)$ and $\tilde{\Omega}_{\beta}(Q, P, t)$ appearing in Eq. (3.3) are obtained from Eqs. (2.2) and (2.3) by substitution of variables. The structure of $K$ is suggested by Theorem 1 and guarantees the stationarity of the hypersurface of the constraints. The extra term $\partial \tilde{\Omega}_{\beta} / \partial t$ is due to the explicit dependence on $t$ of the canonical transformation.

Following the usual procedure of standard classical mechanics we will prove the following theorem:

Theorem 2: Let Eq. (2.18) be the equations of motion of a dynamical system; a transformation

$$
Q_{s}=Q_{s}(q, p, t), \quad P_{s}=P_{s}(q, p, t),
$$

for which two functions $K_{c}$ and $F$ exist so that

$$
p_{s} \delta q_{s}-H_{c} \delta t=P_{s} \delta Q_{s}-K_{c} \delta t-\delta F
$$

is canonical.

Proof: From Eq. (3.5) we have

$$
\oint_{C}\left[p_{s} \delta q_{s}-H_{c} \delta t-\left(P_{s} \delta Q_{s}-K_{c} \delta t\right)\right]=0,
$$

where $C$ is an arbitrary closed contour in the extended phase space, that we will take lying on $S$. Let $\tilde{C}$ be the contour obtained from $C$ by means of the transformation (3.4). Then the Poincaré-Cartan integral is invariant under the considered transformation. In fact, from Eq. (3.6) we get

$$
\oint_{C}\left(p_{s} \delta q_{s}-H_{c} \delta t\right)=\oint_{\bar{C}}\left(P_{s} \delta Q_{s}-K_{c} \delta t\right) .
$$

The left-hand side of Eq. (3.7) is invariant under displacement of the contour along the tube of the dynamical trajectories, solutions of Eq. (2.18) and lying on $S$. The righthand side will be invariant under displacement of the contour $\tilde{C}$ along the tube obtained by means of the transformation (3.4) from the proceeding. On the other hand, the transformed trajectories obey a system of first order differential equations. Thus, by repeating the proof of Theorem 1 and by taking into account the explicit dependence of the constraints on the time, we get

$$
\dot{Q_{s}} \approx \frac{\partial K}{\partial P_{s}}, \quad \dot{P}_{s} \approx-\frac{\partial K}{\partial Q_{s}},
$$

with $K$ given by Eq. (3.3), and therefore the transformation is canonical.

\section{A SET OF CANONICAL VARIABLES INDEPENDENT WITH RESPECT TO THE SECOND-CLASS CONSTRAINTS}

In Ref. 7, as we have reviewed in Sec. 2, the Hamilton equations for a constrained system have been obtained [Eq. (2.18)]. The variables $q_{s}$ and $p_{s}$ are not independent, since they must satisfy Eqs. (2.2) and (2.3). A suitable method for isolating the true independent variables has been developed by Shanmugadhasan. ${ }^{3}$ His theory is based on two theorems on function groups ${ }^{6,9}$ and involutory systems $s^{4,5}$ that we recall without giving proofs.

Theorem 3: A noncommutative function group $G$ of rank $r$ is a subgroup of a group of rank $2 n$ whose basis $\left(\phi_{1}, \ldots, \phi_{n}, \psi_{1}, \ldots, \psi_{n}\right)$ can be chosen so that

$$
\left\{\phi_{i}, \phi_{j}\right\}=\left\{\psi_{i}, \psi_{j}\right\}=0, \quad\left\{\psi_{j}, \phi_{i}\right\}=\delta_{i j}, \quad(i, j=1, \ldots, n) .
$$

Theorem 4: A system of $2 m+q$ independent equations (defining a surface $S_{D}$ of dimension $D=2 n-2 m-q$ )

$$
\Omega_{\tau}=0 \quad(\tau=1, \ldots, 2 m+q),
$$

such that

$$
\operatorname{rank}\left\|\left\{\Omega_{\sigma}, \Omega_{\sigma^{\prime}}\right\}\right\|=2 m, \quad\left(\sigma, \sigma^{\prime}=1, \ldots, 2 m+q\right),
$$

can be substituted by a locally equivalent system

$$
\begin{aligned}
& \phi_{\lambda}=0 \quad(\lambda=1, \ldots, m+q), \\
& \psi_{\alpha}=0 \quad(\alpha=1, \ldots, m),
\end{aligned}
$$

for which the relations

$$
\begin{aligned}
& \left\{\phi_{\lambda}, \phi_{\mu}\right\}=\left\{\psi_{\alpha}, \psi_{\beta}\right\}=0, \\
& \left\{\psi_{\alpha}, \phi_{\lambda}\right\}=\delta_{\alpha \lambda}
\end{aligned}
$$

hold locally in the phase space.

First let us apply the last theorem to the set of secondclass constraints ${ }^{10}$ [Eqs. (2.3)]. Let

$$
Q_{f}=0, \quad P_{f}=0, \quad\left(f=n_{2}+1, \ldots, n\right),
$$

$\left(n_{2}=n-W / 2\right)$ be the locally equivalent system such that

$$
\left\{Q_{f}, P_{f^{\prime}}\right\}=\delta_{f f^{\prime}}, \quad\left\{Q_{f}, Q_{f^{\prime}}\right\}=\left\{P_{f}, P_{f^{\prime}}\right\}=0 \text {. }
$$

The set $G=\left\{Q_{f}, P_{f}, f=n_{2}+1, \ldots, n\right\}$ now forms a noncommutative function group. Theorem 3 enables us to find a $2 n$ dimensional function group which contains $G$. Let

$$
\begin{aligned}
& \left\{Q_{s}^{\prime}, P_{s}^{\prime} ; s=1, \ldots, n\right\} \\
& \quad \equiv\left\{Q_{j}^{\prime}, P_{j}^{\prime}, Q_{f}, P_{f} ; j=1, \ldots, n_{2}, f=n_{2}+1, \ldots, n\right\}
\end{aligned}
$$

denote this function group. Due to the equations

$$
\left\{Q_{s}^{\prime}, P_{s^{\prime}}^{\prime}\right\}=\delta_{s s^{\prime}}, \quad\left\{Q_{s}^{\prime}, Q_{s^{\prime}}^{\prime}\right\}=\left\{P_{s}^{\prime}, P_{s^{\prime}}^{\prime}\right\}=0,
$$

and denoting the new Hamitonian by $K_{\mathrm{c}}$ and the generating function by $F$, we will have, in the usual way, ${ }^{8}$

$$
p_{s} \delta q_{s}-H_{c} \delta t=P_{s}^{\prime} \delta Q_{s}^{\prime}-K_{c} \delta t-\delta F .
$$


We can now apply Theorem 2 and deduce that the transformation

$$
q_{s}, p_{s}, t \rightarrow Q_{s}^{\prime}, P_{s}^{\prime}, t
$$

is canonical. The equations of motion (3.2) become

$$
\begin{aligned}
& \dot{Q}_{j} \approx\left\{Q_{j}, K\right\}, \quad \dot{P}_{j}^{\prime} \approx\left\{P_{j}, K\right\} \quad\left(j=1, \ldots, n_{2}\right) \\
& \dot{Q}_{f} \approx\left\{Q_{f}, K\right\}, \quad \dot{P}_{f} \approx\left\{P_{f}, K\right\} \quad\left(f=n_{2}+1, \ldots, n\right),
\end{aligned}
$$

with

$K=K_{c}+l_{\alpha} \tilde{\Omega}_{\alpha}-\tilde{\Omega}_{\beta} \tilde{c}_{\beta \beta^{\prime}}\left[\left\{\tilde{\Omega}_{\beta^{\prime}}, K_{c}\right\}+\frac{\partial \tilde{\Omega}_{\beta^{\prime}}}{\partial t}\right]$

where

$$
\tilde{\Omega}_{\alpha, \beta}\left(Q_{s}^{\prime}, P_{s}^{\prime}, t\right)=\Omega_{\alpha_{, \beta}}\left[q_{s}\left(Q_{s}^{\prime} P_{s}^{\prime} t\right), p_{s}\left(Q_{s}^{\prime} P_{s}^{\prime} t\right)\right]
$$

are the expressions of the constraints in the new variables.

The term $\partial \tilde{\Omega}_{\beta} / \partial t$ in the Hamiltonian (4.14) can be removed as a consequence of the stationarity of the hypersurface.

In fact, since when $Q_{f}=P_{f}=0$ we have $\tilde{\Omega}_{\beta}=0$, we can develop $\tilde{\Omega}_{\beta}$ in a power series of $Q_{f}$ and $P_{f}$, i.e.,

$\tilde{\Omega}_{\beta}\left(Q_{s}^{\prime}, P_{s}^{\prime}, t\right)$ $=a_{\beta}^{f}\left(Q_{j}^{\prime}, P_{j}^{\prime}, t\right) Q_{f}+b_{\beta}^{f}\left(Q_{j}^{\prime}, P_{j}^{\prime}, t\right) P_{f}+$ higher orders.

If we introduce, following Sudarshan and Mukunda, ${ }^{2}$ the notation of "strong" equality (), we can rewrite Eq. (4.16) as

$$
\tilde{\Omega}_{\beta}\left(Q_{s}^{\prime}, P_{s}^{\prime}, t\right) \equiv a_{\beta}^{f}\left(Q_{j}^{\prime}, P_{j}^{\prime}, t\right) Q_{f}+b_{\beta}^{f}\left(Q_{j}^{\prime}, P_{j}^{\prime}, t\right) P_{f} .
$$

From Eq. (4.17) we can also locally get the inverse relations

$$
\left\{\begin{array}{l}
Q_{f} \equiv \tilde{c}_{f}^{\beta}\left(Q_{j}^{\prime}, P_{j}^{\prime}, t\right) \tilde{\Omega}_{\beta} \\
P_{f} \equiv \tilde{d}_{f}^{\beta}\left(Q_{j}^{\prime}, P_{j}^{\prime}, t\right) \tilde{\Omega}_{\beta},
\end{array}\right.
$$

and in terms of the old variables

$$
\left\{\begin{array}{l}
Q_{f}\left(q_{s}, p_{s}, t\right) \equiv c_{f}^{\beta}\left(q_{s}, p_{s}, t\right) \Omega_{\beta}\left(q_{s}, p_{s}\right) \\
P_{f}\left(q_{s}, p_{s}, t\right) \equiv d_{f}^{\beta}\left(q_{s}, p_{s}, t\right) \Omega_{\beta}\left(q_{s}, p_{s}\right)
\end{array}\right.
$$

By taking the partial derivative with respect to $t$ of Eqs. (4.19) we get

$$
\frac{\partial Q_{f}}{\partial t} \approx 0 . \quad \frac{\partial P_{f}}{\partial t} \approx 0
$$

On the other hand, if we take the total derivative with respect to $t$ of Eq. (4.19) and use the stationarity of the $\Omega_{\beta}$ 's we have

$$
\frac{d}{d t} Q_{f} \approx 0, \frac{d}{d t} P_{f} \approx 0,
$$

and finally from Eq. (4.17),

$$
\frac{\partial}{\partial t} \tilde{\Omega}_{\beta} \approx 0 \text {. }
$$

Thus the last term of the Hamiltonian can be dropped, because it is strongly equal to zero. Then Eqs. (4.12) and (4.13) become

$$
\left\{\begin{array}{l}
\dot{Q}_{j}^{\prime} \approx\left\{Q_{j}^{\prime}, K_{c}+l_{\alpha} \tilde{\Omega}_{\alpha}-\tilde{\Omega}_{\beta} \tilde{c}_{\beta B^{\prime}}\left\{\tilde{\Omega}_{\beta^{\prime}}, K_{c}\right\}\right\}, \\
\dot{P}_{j} \approx\left\{P_{j}, K_{\mathrm{c}}+l_{\alpha} \tilde{\Omega}_{a}-\tilde{\Omega}_{\beta} \tilde{c}_{\beta B^{\prime}}\left\{\tilde{\Omega}_{\beta^{\prime}}, K_{c}\right\}\right\},
\end{array}\right.
$$

$$
\left\{\begin{array}{l}
\dot{Q}_{f} \approx\left\{Q_{f}, K_{c}+l_{\alpha} \tilde{\Omega}_{\alpha}-\tilde{\Omega}_{\beta} \tilde{c}_{\beta \beta^{\prime}}\left\{\tilde{\Omega}_{\beta^{\prime}}, K_{c}\right\}\right\} \\
\dot{P}_{f} \approx\left\{P_{f}, K_{\mathrm{c}}+l_{\alpha} \tilde{\Omega}_{\alpha}-\tilde{\Omega}_{\beta} \tilde{c}_{\beta \beta^{\prime}}\left\{\tilde{\Omega}_{\beta^{\prime}}, \boldsymbol{K}_{c}\right\}\right\}
\end{array}\right.
$$

or, using Eqs. (4.17) and (4.18),

$$
\begin{aligned}
& \left\{\begin{array}{l}
\dot{Q}_{j}^{\prime} \approx\left\{Q_{j}^{\prime}, K_{c}+l_{\alpha} \tilde{\Omega}_{\alpha}\right\}=\left\{Q_{j}^{\prime}, \tilde{K}\right\} \\
\dot{P}_{j}^{\prime} \approx\left\{P_{j}^{\prime}, K_{c}+l_{\alpha} \tilde{\Omega}_{\alpha}\right\}=\left\{P_{j}^{\prime}, \tilde{K}\right\}
\end{array} \quad\left(j=1, \ldots, n_{2}\right),\right. \\
& \left\{\begin{array}{l}
\dot{Q}_{f} \approx 0 \\
\dot{P}_{f} \approx 0
\end{array}\left(f=n_{2}+1, \ldots, n\right)\right.
\end{aligned}
$$

where the last equalities of Eq. (4.25) define $\tilde{K}$.

If we denote the set of variables which are independent with respect to second-class constraints by $R$

$$
R \equiv\left\{Q_{j}^{\prime}, P_{j}^{\prime} ; j=1, \ldots, n_{2}\right\},
$$

the equations of motion, in this reduced phase space, can be rewritten as

$$
\left\{\begin{array}{l}
\dot{Q}_{j}^{\prime} \approx\left\{Q_{j}^{\prime}, \tilde{K}\right\}_{R} \approx\left\{Q_{j}^{\prime}, \bar{K}\right\}_{R}, \\
\dot{P}_{j}^{\prime} \approx\left\{P_{j}^{\prime}, \tilde{K}\right\}_{R} \approx\left\{P_{j}^{\prime}, \bar{K}\right\}_{R},
\end{array}\right.
$$

where $\{,\}_{R}$ are the Poisson brackets defined in the space $R$ and

$$
\bar{K}=\bar{K}_{\mathrm{c}}+l_{\alpha} \bar{\Omega}_{\alpha},
$$

with $\bar{K}_{c}$ and $\bar{\Omega}_{\alpha}$ obtained by setting to zero the variables $Q_{f}$ and $P_{f}$ in $K_{c}$ and $\tilde{\Omega}_{\alpha}$. The "weak" equalities of Eq. (4.28) are equalities on the surface determined by

$$
\bar{\Omega}_{\alpha}\left(Q_{j}^{\prime}, P_{j}^{\prime}\right)=0 \text {. }
$$

Let us observe that, since $\Omega_{\alpha}$ are first-class, we have also

$$
\begin{aligned}
& \left\{\tilde{\boldsymbol{\Omega}}_{\alpha}, \tilde{\Omega}_{\alpha^{\prime}}\right\} \approx 0, \\
& \left\{\tilde{\boldsymbol{\Omega}}_{\alpha}, \tilde{\Omega}_{\beta}\right\} \approx 0 .
\end{aligned}
$$

From Eqs. (4.18) and (4.32) we get

$\frac{\partial \tilde{\Omega}_{\alpha}}{\partial P_{f}}=\left\{Q_{f}, \tilde{\Omega}_{\alpha}\right\} \approx 0, \frac{\partial \tilde{\Omega}_{\alpha}}{\partial Q_{f}}=-\left\{P_{f}, \tilde{\Omega}_{\alpha}\right\} \approx 0$.

Therefore, by defining

$$
\begin{aligned}
\left\{\tilde{\Omega}_{\alpha}, \tilde{\Omega}_{\alpha^{\prime}}\right\}_{R}= & \left\{\tilde{\Omega}_{\alpha}, \tilde{\Omega}_{\alpha^{\prime}}\right\}-\left(\frac{\partial \tilde{\Omega}_{\alpha}}{\partial Q_{f}} \frac{\partial \tilde{\Omega}_{\alpha^{\prime}}}{\partial P_{f}}\right. \\
& \left.-\frac{\partial \tilde{\Omega}_{\alpha}}{\partial P_{f}} \frac{\partial \tilde{\Omega}_{\alpha^{\prime}}}{\partial Q_{f}}\right),
\end{aligned}
$$

and using Eqs. (4.33) and (4.31), we have

$$
\left\{\tilde{\Omega}_{\alpha}, \tilde{\Omega}_{\alpha^{\prime}}\right\}_{R} \approx 0 \text {, }
$$

which also implies

$$
\left\{\bar{\Omega}_{\alpha}, \bar{\Omega}_{\alpha^{\prime}}\right\}_{R} \approx 0 \text {. }
$$

Finally let us prove that the hypersurface determined by Eq. (4.30) is stationary.

In fact,

$$
\begin{aligned}
\frac{d}{d t} \bar{\Omega}_{\alpha} & \approx\left\{\bar{\Omega}_{\alpha}, \bar{K}\right\}_{R} \approx\left\{\bar{\Omega}_{\alpha}, \bar{K}_{c}\right\}_{R} \\
& \approx\left\{\tilde{\Omega}_{\alpha}, K_{c}\right\}_{R} \approx\left\{\bar{\Omega}_{\alpha}, K_{c}\right\},
\end{aligned}
$$

where use was made of Eqs. (4.33) and (4.36). On the other hand, due to the canonical character of the transformation (4.11), Eqs. (2.7) imply

$$
\left\{\tilde{\Omega}_{\alpha}, K_{c}\right\} \approx 0 \text {, }
$$


and thus

$$
\frac{d}{d t} \bar{\Omega}_{\alpha} \approx\left\{\overline{\boldsymbol{\Omega}}_{\alpha}, \overline{\boldsymbol{K}}_{c}\right\}_{R} \approx 0
$$

Summing up, we have shown that it is possible, by making use of a canonical transformation, to write the equations of motion for a set of variables which are independent with respect to second-class constraints. As shown in Ref. 3, we have the following relation between Dirac brackets and Poisson brackets defined in the reduced space $R$ :

$$
\{,\}^{*}=\{,\}_{R} \text {. }
$$

Therefore the variables which are canonical with respect to Dirac brackets are directly obtained by means of this canonical transformation.

\section{EQUATIONS OF MOTION FOR A SET OF UNCONSTRAINED VARIABLES}

A further step can be done by extending the transformation to include first-class constraints too.

In fact, Theorem 4 guarantees that it is always possible, at least locally, to replace the $\bar{\Omega}_{\alpha}$ by an equivalent set

$$
P_{e}=0, \quad\left(e=n_{1}+1, \ldots, n_{2}\right),
$$

$\left(n_{1}=n-T+W / 2\right) ;$ such that the equations

$$
\left\{P_{e}, P_{e^{\prime}}\right\}=0
$$

are identically satisfied and not by virtue of Eqs. (5.1) themselves. The same theorem shows that the $P_{e}$ can be obtained by solving Eq. (4.30) for $n_{2}-n_{1}$ of the momenta $P_{j}^{\prime}$ in terms of the remaining momenta and of the coordinates $Q_{j}^{\prime}$. We can, by renumbering the variables if necessary, assume that Eq. (4.30) can be solved for the last $n_{2}-n_{1} P_{j}^{\prime}$ in terms of the first $n_{1} P_{j}^{\prime}$ and all the $Q_{j}^{\prime}$, i.e.,

$$
\begin{aligned}
P_{c}=P_{c}^{\prime}-f_{e}\left(Q_{e}^{\prime}, Q_{k}^{\prime}, P_{k}^{\prime}\right) \quad\left(k=1, \ldots, n_{1}\right) \\
\left(e=n_{1}+1, \ldots, n_{2}\right) .
\end{aligned}
$$

We observe, from Eq. (5.3), the local character of this technique. Thus, in general, we will have to repeat the procedure we will develop in the following, for the different sheets of the hypersurface (4.30).

Let us notice that from Eqs. (4.9) and (5.3) we have

$$
\left\{Q_{e}^{\prime}, P_{\mathfrak{e}^{\prime}}\right\}_{R}=\delta_{e e^{\prime}} \text {, }
$$

or, following the terminology of the function groups, $Q_{e}^{\prime}$ (that from now on we will call $Q_{e}$ ) and $P_{e}$ form a noncommutative function group of dimension $2\left(n_{2}-n_{1}\right)$. By applying again Theorem 3 we can construct a canonical transformation

$Q_{j}^{\prime}, P_{j}^{\prime}, t \rightarrow Q_{k}, P_{k}, Q_{e}, P_{e} \quad\left(k=1, \ldots, n_{1}\right),\left(e=n_{1}+1, \ldots, n_{2}\right)$,

with

$$
\left\{Q_{k}, P_{k^{\prime}}\right\}=\delta_{k k^{\prime}}, \quad\left\{Q_{e}, P_{e^{\prime}}\right\}=\delta_{e e^{\prime}},
$$

and the other Poisson brackets vanishing.

If we denote the new canonical Hamiltonian by $\hat{K}_{c}$, and if we write the constraints (4.30) in terms of the new variables as

$$
\begin{aligned}
& \hat{\Omega}\left(Q_{k}, P_{k}, Q_{e}, P_{e}\right) \\
& \quad=\bar{\Omega}_{\alpha}\left(Q_{j}^{\prime}\left(Q_{k}, P_{k}, Q_{e}, P_{e}\right), P_{j}^{\prime}\left(Q_{k}, P_{k}, Q_{e}, P_{e}\right)\right)=0,
\end{aligned}
$$

the new equations of motion can be obtained by applying Theorem 2 in the reduced phase space $R$ :

$$
\left\{\begin{array}{l}
\dot{Q}_{k} \approx\left\{Q_{k}, \hat{K}_{c}+l_{\alpha} \hat{\Omega}_{\alpha}\right\}_{R}, \\
\dot{P}_{k} \approx\left\{P_{k}, \hat{K}_{c}+l_{\alpha} \hat{\Omega}_{\alpha}\right\}_{R},
\end{array}\right.
$$

and

$$
\left\{\begin{array}{l}
\dot{Q}_{e} \approx\left\{Q_{e}, \hat{K}_{c}+l_{\alpha} \hat{\Omega}_{\alpha}\right\}_{R}, \\
\dot{P}_{e} \approx\left\{P_{e}, \hat{K}_{c}+l_{\alpha} \hat{\Omega}_{\alpha}\right\}_{R} .
\end{array}\right.
$$

On the other hand, due to the fact that when $P_{e}=0, \hat{\Omega}_{\alpha}$ $=0$, we can write, as for the second-class constraints [Eqs. (4.17)],

$$
\hat{\Omega}_{\alpha}\left(Q_{k}, P_{k}, Q_{e}, P_{e}\right) \equiv g_{\alpha}^{e}\left(Q_{k}, P_{k}, Q_{e}\right) P_{e} .
$$

Thus substituting Eq. (5.10) in Eq. (5.8) and (5.9), we get

$$
\begin{aligned}
& \left\{\begin{array}{l}
\dot{Q}_{k} \approx\left\{Q_{k}, \hat{K}_{c}+\lambda_{e^{\prime}} P_{e^{\prime}}\right\}_{R} \approx\left\{Q_{k}, \hat{K}_{c}\right\}_{R}, \\
\dot{P}_{k} \approx\left\{P_{k}, \hat{K}_{c}+\lambda_{e^{\prime}} P_{e^{\prime}}\right\}_{R} \approx\left\{P_{k}, \hat{K}_{c}\right\}_{R},
\end{array}\right. \\
& \left\{\begin{array}{l}
\dot{Q}_{e} \approx\left\{Q_{e}, \hat{K}_{c}+\lambda_{e^{\prime}} P_{e^{\prime}}\right\}_{R} \approx\left\{Q_{e}, \hat{K}_{c}\right\}_{R}+\lambda_{e}, \\
\dot{P}_{e} \approx\left\{P_{e}, \hat{K}_{c}+\lambda_{e^{\prime}} P_{e^{\prime}}\right\}_{R} \approx\left\{P_{e}, \hat{K}_{c}\right\}_{R},
\end{array}\right.
\end{aligned}
$$

where $\lambda_{e}=l_{\alpha} g_{\alpha}^{e}$ are arbitrary functions of $t$.

Let us finally show that this sheet of hypersurface is stationary. By differentiating Eq. (4.30) with respect to any variable $u\left(Q_{j}^{\prime}\right.$ or $\left.P_{j}^{\prime}\right)$ and using Eq. (5.3) we get

$$
\frac{\partial \bar{\Omega}_{\alpha}}{\partial u}=-\frac{\partial \bar{\Omega}_{\alpha}}{\partial P_{e}^{\prime}} \frac{\partial f_{e}}{\partial u}=\frac{\partial \bar{\Omega}_{\alpha}}{\partial P_{e}^{\prime}} \frac{\partial P_{e}}{\partial u} .
$$

Therefore

$$
\left\{\bar{\Omega}_{\alpha}, \bar{K}_{c}\right\}_{R}=\frac{\partial \bar{\Omega}_{a}}{\partial P_{c}^{\prime}}\left\{P_{c}, \bar{K}_{c}\right\}_{R},
$$

and using Eq. (4.39) and the fact that we locally have

$$
\operatorname{det}\left|\frac{\partial \bar{\Omega}_{\alpha}}{\partial P_{e}^{\prime}}\right| \neq 0,
$$

we get

$$
\left\{P_{e}, \bar{K}_{c}\right\}_{R} \approx 0,
$$

and after the canonical transformation (5.5)

$$
\frac{\partial \hat{K}_{c}}{\partial Q_{e}}=\left\{\hat{K}_{c}, P_{e}\right\}_{R} \approx 0,
$$

which ensures the hypersurface be stationary. In addition Eq. (5.17) state that the variables $Q_{e}$ are ignorable variables on the hypersurface. ${ }^{3}$

Thus we have the following equations of motion:

$$
\begin{aligned}
& \dot{Q}_{k} \approx\left\{Q_{k}, \hat{K}_{c}\right\}_{R} \quad \dot{P}_{k} \approx\left\{P_{k}, \hat{K}_{c}\right\}_{R}, \\
& \dot{Q}_{c} \approx\left\{Q_{e}, \hat{K}_{c}\right\}+\lambda_{e} \quad \dot{P}_{e} \approx 0,
\end{aligned}
$$

where $\lambda_{e}$ are arbitrary functions.

We can finally consider a reduced space of unconstrained variables $\left[Q_{k}, P_{k}\right.$ and $\left.Q_{e}\right]$, which are the intrinisic coordinates of the hypersurface of the motion:

$$
Q_{f}=0, \quad P_{f}=0, \quad P_{c}=0 .
$$


Their evolution equations are

$$
\begin{aligned}
& \dot{Q}_{k}=\left.\frac{\partial \hat{K}_{c}}{\partial P_{k}}\right|_{P_{e}=0}, \quad \dot{P}_{k}=-\left.\frac{\partial \hat{K}_{c}}{\partial Q_{k}}\right|_{P_{e}=0}, \\
& \dot{Q}_{e}=\left.\frac{\partial \hat{K}_{c}}{\partial P_{e}}\right|_{P_{e}=0}+\lambda_{e} .
\end{aligned}
$$

If we put

$$
\mathscr{K}_{c}\left(Q_{k}, P_{k}, t\right)=\left.\hat{K}_{c}\left(Q_{k}, P_{k}, Q_{e}, P_{e}, t\right)\right|_{P_{v}=0},
$$

Eqs. (5.20) can be rewritten as

$$
\begin{aligned}
& \dot{Q}_{k}=\frac{\partial \mathscr{K}_{c}}{\partial P_{k}}\left(Q_{k}, P_{k}, t\right), \\
& \dot{P}_{k}=-\frac{\partial \mathscr{K}_{c}}{\partial Q_{k}}\left(Q_{k}, P_{k}, t\right) \quad\left(k=1, \ldots, n_{1}\right),
\end{aligned}
$$

whereas Eqs. (5.21) are left unchanged since the two operations of setting $P_{e}=0$ and differentiating with respect to $P_{e}$ do not commute.

As shown by Eqs. (5.20) and (5.21) this method allows to isolate the gauge independent variables $Q_{k}, P_{k}$ (physical variables) from the gauge dependent $Q_{e}$, whose evolution is determined only when the arbitrary functions are given.

We point out that when $H_{\mathrm{c}}=0$, that is when, if a lagrangian formulation exists, the action is parameter-invariant, ${ }^{11}$ we have $\hat{K}_{c}=0$ and Eqs. (5.20) and (5.21) become

$$
\begin{aligned}
& \dot{Q}_{k}=0, \quad \dot{P}_{k}=0, \\
& \dot{Q}_{e}=\lambda_{e} .
\end{aligned}
$$

Thus, for what concerns the physical variables, the procedure is equivalent to the Hamilton-Jacobi method.

Let us finally observe that we can choose anyone of the coordinates $Q_{e},{ }^{12}$ for example $Q_{\bar{e}}\left(n_{1}<\bar{e} \leqslant n_{2}\right)$, as evolution parameter and rewrite Eqs. (5.24) and (5.25) as

$$
\begin{aligned}
& \frac{d Q_{k}}{d Q_{\bar{e}}}=0, \quad \frac{d P_{k}}{d Q_{\bar{e}}}=0, \\
& \frac{d Q_{r}}{d Q_{\bar{e}}}=\lambda_{r} / \lambda_{\bar{e}} \quad\left(r=n_{1}+1, \ldots \bar{e}-1, \bar{e}+1, \ldots n_{2}\right) \\
& \dot{Q}_{\bar{e}}=\lambda_{\bar{e}} .
\end{aligned}
$$

Thus in order to get the relation between $Q_{r}$ and $Q_{\bar{e}}$ we must give the ratio of the arbitrary functions $\lambda_{r} / \lambda_{\bar{e}}$ as a function of $Q_{\bar{e}}$ and if we are interested in the relation between $Q_{\bar{e}}$ and the unphysical parameter $t$ we must give $\lambda_{\bar{e}}$ as a function of $t$.

Therefore, with this procedure we get a reduced class of gauge (we can only choose one of the coordinates $Q_{e}$ as evolution parameter). This a consequence of the definition of $P_{e}$ [Eqs. (5.3)]. On the other hand, different classes of gauges can be obtained by solving Eq. (4.30) to a different set of momenta.

\section{CONCLUSIONS}

Making use of the Poincaré-Cartan integral for constrained systems, we have shown that the invariance of this integral enables us to write the equations of motion for a dynamical system as Hamilton equations. We want to observe that with this procedure, all the first-class constraints appear in the Hamiltonian, because we cannot introduce any distinction between them. Recent papers, by Cawley ${ }^{13}$ and Frenkel, ${ }^{14}$ have shown, with some examples, that not all the first-class secondary constraints generate gauge transformations and therefore not all the first-class constraints appear in the Hamiltonian. ${ }^{15}$ Thus we are investigating an algebraic procedure in order to take into account this result.

Furthermore, we have introduced a definition of canonical transformation, which is the trivial generalization of the usual one, and shown that the Poincaré-Cartan integral is invariant under this transformation. Then, following Shanmugadhasan, ${ }^{3}$ we have performed a canonical transformation such that a subset of the new variables is equivalent to the second-class constraints. The reduced set of variables, independent with respect to second-class constraints, is nothing but the set of variables which are canonical with respect to Dirac brackets.

A further step is done by performing a new canonical transformation in the reduced phase space which isolates the variables corresponding to first-class constraints. This transformation is very useful because it isolates also the gauge independent variables from the gauge dependent ones. The evolution of these gauge dependent variables, contrary to the result of Shanmugadhasan, consistently depends on arbitrary functions.

When $H_{c}=0$ this technique becomes equivalent to the Hamilton-Jacobi method. Explicit examples (the free relativistic point and a model of two interacting relativistic particles) have been already studied ${ }^{16}$; presently we are investigating the possibility of extending this technique to continuous systems, studying the relativistic string model (see Nambu and Scherk in Ref. 1).

\section{ACKNOWLEDGMENTS}

We wish to thank Dr. L. Lusanna of the "Istituto Nazionale di Fisica Nucleare" of Florence for many stimulating and interesting discussions.

\footnotetext{
'Y. Nambu, "Lectures for the Copenhagen Summer Symposium 1970" (unpublished); J. Scherk, Rev. Mod. Phys. 47, 123 (1975); R. Casalbuoni and G. Longhi, Nuovo Cimento A 25, 482 (1975); M. Kalb and P. Van Alstine, "Invariant Singular Actions for the Relativistic Two Body Problem: a Hamiltonian Formulation," Yale report C00-3075-146 (June 1976); T. Takabayasi and S. Kojima, Prog. Theor. Phys. 57, 2127 (1977); D. Dominici, J. Gomis and G. Longhi, Nuovo Cimento B 48, 152 (1978); Nuovo Cimento A 48, 257 (1978)

${ }^{2}$ P. A. M. Dirac, "Lectures on Quantum Mechanics," Belfer Graduate School of Science, Yeshiva University (New York, 1964); for a comprehensive exposition of the Dirac theory see also E. C. G. Sudarshan and N. Mukunda, Classical Dynamics: a Modern Perspective (Wiley, New York, 1974).

${ }^{3}$ S. Shanmugadhasan, J. Math. Phys. 14, 677 (1973).

${ }^{4} \mathrm{~J}$. A. Shouten and W. v. der Kulk, Pfaff's Problem and Its Generalizations (Clarendon, Oxford, 1949).

${ }^{5} \mathrm{C}$. R. Chester, Techniques in Partial Differential Equations, Chap. XV (McGraw-Hill Kogakusha, Tokyo, 1971).

${ }^{6}$ L. P. Eisenhart, Continuous Groups of Transformations, (Dover, New York, 1961).

${ }^{7} \mathrm{~F}$. Benavent and J. Gomis, "Poincaré-Cartan Integral Invariant for Constrained Systems," Ann. Phys. 118, 476 (1979).

${ }^{8} \mathrm{~F}$. Gantmacher, Lectures in Analytical Mechanics (Mir. Moscow, 1970).
} 
${ }^{9} T$ The set $G$ of the functions of $r$ independent functions $F_{1}\left(q_{s}, p_{s}\right) \cdots F_{s}\left(q_{s}, p_{s}\right)$, $(s=1, \ldots, n)$, such that $\left\{F_{a}, F_{b}\right\}=\phi\left(F_{\mathrm{c}}\right),(a, b, c,=1, \ldots, r)$ is said to be a function group of rank $r$. The function group is said commutative if $\left\{F_{a}, F_{b}\right\}=0$ for all the values of $a$ and $b$.

${ }^{10} \mathrm{~L}$. Castellani, D. Dominici, and G. Longhi, Nuovo Cimento A 48, 91 (1978).

"H. Rund, The Hamilton-Jacobi Theory in the Calculus of Variations (Van Nostrand, London, 1966)

${ }^{12} \mathrm{Also}$ in Ref. 7 we have introduced as evolution parameter one of the coordinates $q_{*}$, but in that case the coordinates are not independent with respect to the constraints.

${ }^{13}$ R. Cawley, Phys. Rev. Lett. 42, 413 (1979).

${ }^{14}$ A. Frenkel, "Comment on Cawley's Counter Example to a Conjecture of Dirac," preprint KFK1-1979-45, Central Research Institute for Physics, Budapest, Hungary.

${ }^{15}$ See also M. I. Gotay, "On the Validity of Dirac's Conjecture Regarding First-Class Secondary Constraints," University of Calgary preprint (November 1979) and references quoted therein.

${ }^{16} \mathrm{D}$. Dominici, J. Gomis and G. Longhi, in preparation. 